\title{
A Case of Lichen Planus Pemphigoides Successfully Treated with a Combination of Cyclosporine A and Prednisolone
}

\author{
Ken Washio ${ }^{a}$ Atsuko Nakamura ${ }^{a}$ Shunpei Fukuda ${ }^{b}$ \\ Takashi Hashimoto $^{b}$ Tatsuya Horikawa ${ }^{a}$ \\ ${ }^{a}$ Department of Dermatology, Nishi-Kobe Medical Center, Kobe, and ${ }^{b}$ Department of \\ Dermatology, Kurume University School of Medicine and Kurume University Institute of \\ Cutaneous Cell Biology, Kurume, Japan
}

\section{Key Words}

Lichen planus pemphigoides · Bullous pemphigoid · Lichen planus · Cyclosporine A

\begin{abstract}
Lichen planus pemphigoides (LPP) is a rare clinical variant of bullous pemphigoid (BP). A 35year-old female patient presented to our hospital complaining of pruritic violaceous-colored plaques or papules on the extremities. Tense vesicles were also seen on the soles. Skin biopsies from the papules and vesicles demonstrated lichen planus and BP, respectively. Direct immunofluorescence demonstrated linear IgG and C3 deposition on the basement membrane zone. Indirect immunofluorescence on $1 \mathrm{M} \mathrm{NaCl}$ split skin detected IgG reactivity with the epidermal side. Enzyme-linked immunosorbent assay also detected anti-BP180 antibodies. After treatment with oral prednisolone alone had failed, low-dose cyclosporine A (CyA) was added. The clinical symptoms immediately improved and the titer of the antiBP180 antibodies decreased. Although there is little information about the treatment of recalcitrant LPP, additional CyA appeared to be beneficial.
\end{abstract}

\section{Introduction}

Lichen planus pemphigoides (LPP) is a rare clinical variant of bullous pemphigoid (BP), which is characterized by clinical and histological findings of both lichen planus (LP) and BP. Although several reports of LPP suggest an efficacy of corticosteroid therapy with or without dapsone [1], there is limited information about the treatment of severe corticosteroid-

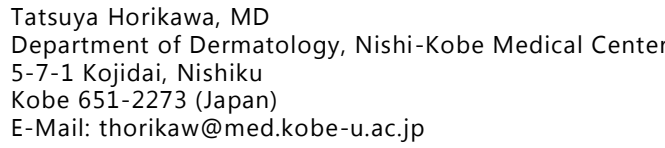


resistant cases of LPP. Hence, we report a case of refractory LPP, which was successfully treated with a combination of low-dose cyclosporine A (CyA) and prednisolone (PSL).

\section{Case Presentation}

A 35-year-old Japanese female was referred to our hospital for itchy skin lesions on the legs. She first noticed pruritic papules in April 2009, and 2 months later many vesicles emerged. On physical examination, flat-topped, polygonal, violaceous-colored plaques or papules were seen on the extremities, and some papules were accompanied by small vesicles or erosions on top of them (fig. 1a). Tense vesicles were also seen on the soles (fig. 1b). Lacelike, reticulated, whitish lesions were seen on the buccal mucosa (fig. 1c). Histopathological examination of the skin biopsy from the papular lesion on the right leg revealed hyperkeratosis, hypergranulosis, acanthosis and liquefaction degeneration in the epidermis and bandlike infiltration of lymphocytes in the upper dermis, which was compatible with LP (fig. 2a). A further biopsy specimen from the right sole showed subepidermal blister formation with moderate inflammatory infiltrates (fig. 2b). Direct immunofluorescence demonstrated linear IgG (fig. 2c) and C3 (fig. 2d) deposition on the basement membrane zone. Indirect immunofluorescence on $1 \mathrm{M} \mathrm{NaCl}$ split skin detected IgG reactivity with the epidermal side (fig. 2e). Reactivity against the recombinant protein of the BP180-NC16a domain, but not the BP180C-terminal domain, was detected by immunoblotting assays (data not shown). Enzymelinked immunosorbent assay also detected anti-BP180 antibodies (index: 39; normal <9). Based on concurrent clinical and pathological features of BP and LP, we diagnosed our case as LPP.

PSL (20 mg/day: $0.4 \mathrm{mg} / \mathrm{kg} /$ day) failed to inhibit disease activity, resulting in exacerbation of vesicle formation, increase of itchiness and elevation of the anti-BP180 antibody titer index to 72. As the patient complained of insomnia caused by low-dose corticosteroid therapy, CyA administration was considered, although an increase in the dose of PSL would have been the standardized therapeutic strategy. Soon after the addition of low-dose CyA (100 mg/day: $2 \mathrm{mg} / \mathrm{kg} /$ day), the vesicles flattened and the itchiness improved. Three months later, the anti-BP180 antibody titer index dropped to 14, and both the skin lesions (fig. 1d, e) and oral enanthemata (fig. 1f) markedly improved. We tapered down the dose of PSL and CyA. The dose of CyA was decreased to $25 \mathrm{mg} /$ day in November 2011, and in January 2013, the dose of PSL was decreased to $2 \mathrm{mg} /$ day. The titer of anti-BP180 antibody has been undetectable since April 2010. The patient maintained remission of bullous formation, although mild itching due to LP persisted. During the follow-up period, the side effect of corticosteroid resulted in a double bone fracture despite bisphosphonate administration. The presumable side effect of combination therapy was influenza, which was successfully treated with oseltamivir. Other serious side effects of CyA, such as sepsis and renal dysfunction, were not observed.

\section{Discussion}

In most cases of LPP, therapy with low to middle doses of oral corticosteroid seems to be effective [1]. In some cases, only topical corticosteroid administration was effective [2]. Combination therapy with corticosteroid and dapsone was also successfully used $[1,3]$. There is little information about combination therapy with immunosuppressant drugs and corticosteroid for LPP. Only one atypical case of LPP with Castleman's disease was treated 
with CyA and mycophenolate mofetil [4]. Despite the absence of previous evidence, CyA therapy appears to have some advantages. First, it does not need hospitalization, which is inevitable for steroid pulsed therapy, intravenous immunoglobulin or plasmapheresis. Second, it is applicable to patients with anemia (as in our case), who cannot be treated with dapsone therapy. Third, CyA inhibits the itching by modulating the function of CD4-positive $\mathrm{T}$ cells. In LPP cases, long-standing LP lesions with basal keratinocyte damage by liquefaction may increase the antigen exposure of the BP180 protein, followed by autoantibody production and induction of bullous lesions [5]. Therefore, it is reasonable to use CyA for the treatment of LPP to suppress the dermal infiltration of lymphocytes in LP lesions. The effectiveness of CyA in our case supports this hypothesis of LPP etiology. Furthermore, another case of severe LP could also be well controlled with a very low dose of CyA [6].

In conclusion, we demonstrated that combination therapy with low-dose CyA and PSL was effective and without any side effects in a case of LPP. Although we reported only one case, this therapy could also be useful in other severe LPP cases.

\section{Disclosure Statement}

The authors have no conflicts of interest to declare.

\section{References}

1 Demirçay Z, Baykal C, Demirkesen C: Lichen planus pemphigoides: report of two cases. Int J Dermatol 2001;40:757-759.

2 Harting MS, Hsu S: Lichen planus pemphigoides: a case report and review of the literature. Dermatol Online J 2006;12:10.

-3 Barnadas MA, Roé E, Dalmau J, Alomar A, Martínez L, Gelpí C: Lichen planus pemphigoides: detection of antiBP 180 antibodies by ELISA and immunoblotting tests. J Eur Acad Dermatol Venereol 2010;24:1360-1361.

-4 Hsiao CJ, Hsu MM, Lee JY, Chen WC, Hsieh WC: Paraneoplastic pemphigus in association with a retroperitoneal Castleman's disease presenting with a lichen planus pemphigoides-like eruption. A case report and review of literature. Br J Dermatol 2001;144:372-376.

5 Stingl G, Holubar K: Coexistence of lichen planus and bullous pemphigoid. A immunopathological study. Br J Dermatol 1975;93:313-320.

6 Levell NJ, Munro CS, Marks JM: Severe lichen planus clears with very low-dose cyclosporin. Br J Dermatol 1992;127:66-67. 


\section{Case Reports in Dermatology}

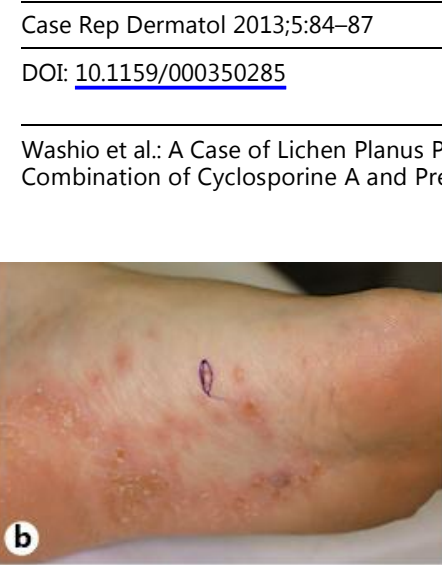

a
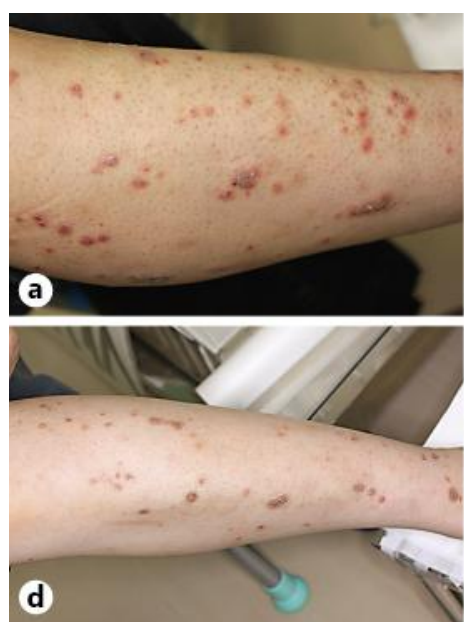

b

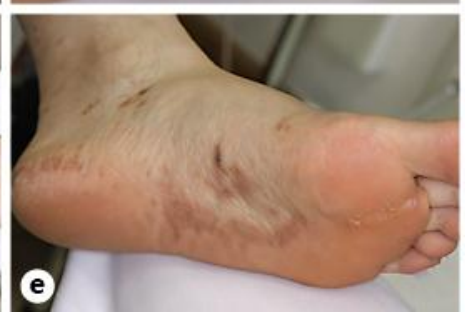

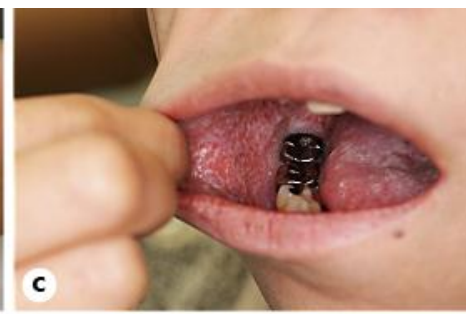

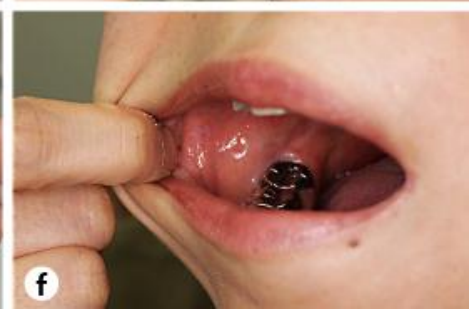

Fig. 1. LP-like lesions on the extremities (a). Vesicles with erythema on the right sole (b). Lace-like lesion on the buccal mucosa (c). After combination therapy with CyA and PSL, these skin and mucosal lesions remarkably improved $(\mathbf{d}-\mathbf{f})$.
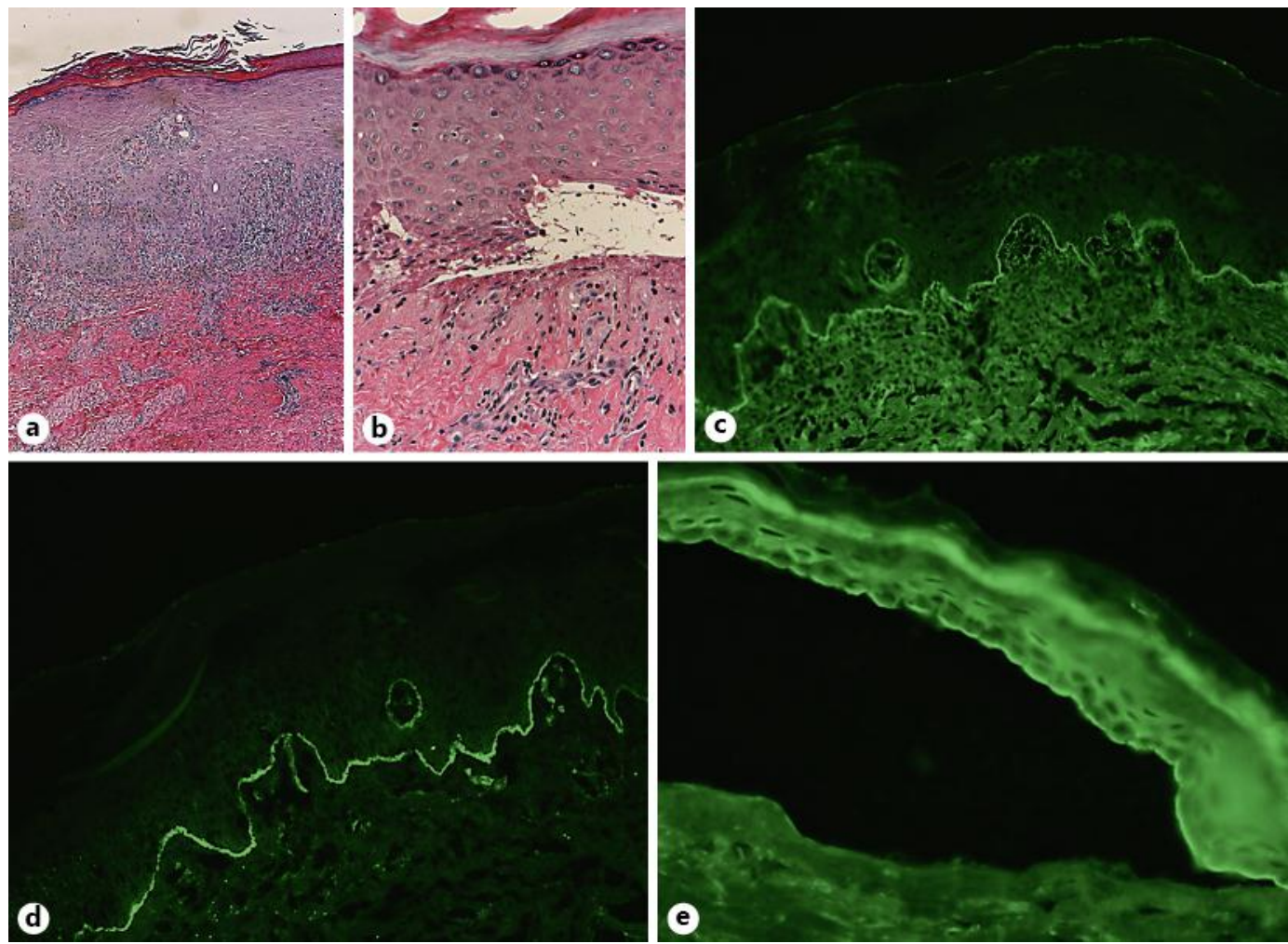

Fig. 2. Histopathological findings of the LP-like $(\mathbf{a} ; \times 40)$ and blistering $(\mathbf{b} ; \times 100)$ skin lesions. The result of direct immunofluorescence for IgG $(\mathbf{c} ; \times 100)$ and C3 $(\mathbf{d} ; \times 100)$. The result of indirect immunofluorescence on $1 \mathrm{M} \mathrm{NaCl}$ split skin for IgG antibodies $(\mathbf{e} ; \times 200)$. 